\title{
Improvement of carrier collection in Si/a-Si:H nanowire solar cells by using
}

\section{hybrid ITO/silver nanowires contacts}

Tiphaine Mathieu-Pennober, ${ }^{1}$ Martin Foldyna, ${ }^{2}$ Shan-Ting Zhang, ${ }^{3,4}$ François H. Julien, ${ }^{1}$ Nathanaelle Schneider, ${ }^{3,4}$ Maria Tchernycheva ${ }^{1}$

1 Centre de Nanosciences et de Nanotechnologies (C2N), UMR 9001 CNRS, Université Paris Saclay, 10

Boulevard Thomas Gobert, 91120 Palaiseau, France.

2 LPICM, CNRS, Ecole Polytechnique, IP Paris, 91128 Palaiseau, France.

3 CNRS, UMR 9006, Institut Photovoltaïque d'lle-de-France (IPVF), 18 boulevard Thomas Gobert, 91120 Palaiseau, France.

4 Institut Photovoltaïque d'Ile-de-France (IPVF), 18 boulevard Thomas Gobert, 91120 Palaiseau, France.

\section{Abstract}

Optoelectronic devices based on high aspect ratio nanowires bring new challenges for transparent electrodes, which can be well addressed by using hybrid structures. Here we demonstrate that a composite contact to radial junction nanowire solar cells made of a thin indium-tin oxide (ITO) layer and silver nanowires greatly improves the collection of charge carriers as compared to a single thick ITO layer by reducing the series resistance losses while improving the transparency. The optimization is performed on $\mathrm{p}$-i-n solar cells comprising of dense non-vertical nanowires with a p-doped c-Si core and an ultra-thin a-Si:H absorption layer grown by plasma-enhanced chemical vapor deposition on glass substrates. The optimal hybrid contact developed in this work is demonstrated to increase the solar cell conversion efficiency from $4.3 \%$ to $6.6 \%$. 


\section{Introduction}

Optoelectronic devices such as touch panels, liquid-crystal displays (LCDs), light-emitting diodes (LEDs) or solar cells require top contact which is both transparent and conductive. For devices with a flat top surface, transparent conductive oxides (TCOs), in particular Sn-doped indium oxide (ITO), have already been optimized and display excellent optical and electronic properties (sheet resistance $R_{\square}$ ca. $10-30 \Omega / \square$ and transmittance at $550 \mathrm{~nm}$ larger than 90\%).(1) However, the most widespread deposition methods for TCOs, among them sputtering, are not well suited for high aspect ratio structures found in nanowire (NW) devices. As sputtering utilizes energetic particles the resulting deposition is directional and the TCO films do not conformally follow the NW morphology.

Semiconductor NW arrays are increasingly used in optoelectronic applications.(2-5) High-quality NWs can be grown on cheap substrates including amorphous ones. $(6,7)$ Thanks to an excellent light trapping and a low optical reflectance, NWs also lead to a better absorption of light with respect to thin films.(8) In c-Si, they have been used either together with planar cells, $(9,10)$ or alone, with top-down and bottom-up approaches.(11-14) One particularity of the NW morphology is that it enables radial junction architecture. It allows to decouple the light absorption and the charge carrier collection as those can be independently improved by the nanowire length and absorber thickness, respectively. This is especially beneficial for hydrogenated amorphous silicon (a-Si:H), which suffers from short minority carrier diffusion lengths.(15)

Despite the potential for a broad use of a-Si:H (non-toxicity, earth abundance, low temperature deposition) compared to other materials for thin-film solar cells, its development remains limited, with a record power conversion efficiency of $10.2 \%$ for a planar cell.(16) When used in NW structure, the absorber layer can be thinner (e.g. $100 \mathrm{~nm}$ in a NW solar cell (17) compared to down to $220 \mathrm{~nm}$ for the record planar cell (16)), which improves the collection of charge carriers with no reduction of the light 
absorption. Heterojunctions with $\mu \mathrm{c}-\mathrm{Si}: \mathrm{H}, \mu \mathrm{c}-\mathrm{SiOx}: \mathrm{H}$ or nc-Si have also been developed,(18-20) with a record conversion efficiency of $9.2 \%$ reported.(21)

In an ideal radial junction solar cell, charge carriers would be collected all along the nanowires with the top contact displaying a low in-plane resistance and a high transmittance. While arrays with sparse, vertical, low aspect-ratio, or tapered nanostructures can be conformally covered using conventional methods (22), new electrodes have to be found when these criteria are not met. Before the deposition of the conductive layer, the high aspect ratio of the structure can be reduced by encapsulation of the NWs in a polymer or by increasing the thickness of the doped layer up to the NW top coalescence.(19) However, this approach compromises some of the advantages for NW solar cells, namely it may increase the reflection and absorption losses and lead to a material waste.

Innovative transparent conductive materials as alternatives to conventional TCOs have been developed. (23-26). Among them, Ag NWs have attracted tremendous attention.(1,27-30) A comparison of experimental results indicates that Ag NWs achieve the best opto-electrical trade-off for a single material. (31) Lagrange et al. showed a sheet resistance as low as $2.9 \Omega / \square$ for $89.2 \%$ transmittance at $550 \mathrm{~nm} .(32)$ Moreover, Ag NWs can be easily deposited with various solution processes.(27-29)

The conductivity of Ag NWs depends on their length and diameter and their density in the network.(33) Because of their very high aspect ratio ( $>150$ in our work), Ag nanowires are prone to connect to form conductive paths. Densities around 4-10 $\mathrm{gg} / \mathrm{cm}^{2}$ for NWs ca. $100 \mathrm{~nm} \times 10 \mu \mathrm{m}$ are required for sheet resistances below $50 \Omega / \square .(1,33,34)$

Although Ag NWs provide a low resistance on a large scale, alone they can hardly work as an effective top contact when applied to a dense array of semiconductor NWs. In fact, an unreasonably high density of Ag NWs would be required to connect all the semiconductor NWs, which would give rise to a significant loss of transparency. 
Instead of using a single material for the top contact, a hybrid electrode can be used. A rich variety of structures with tunable properties have been explored and demonstrated: Ag NWs/graphene,(35,36) TCO/Ag thin film,(37,38) polymer/Ag NWs,(39) TCO/graphene,(40) TCO/Ag NWs,(41-44) etc. Although these contacts have been initially developed for planar devices, some of them are particularly suited to high aspect ratio structures. Recently a few studies have demonstrated the promising potential of using a hybrid contact for NW based optoelectronic devices.(34,45)

In this paper, we report on the optimization of a hybrid contact made of ITO and Ag NWs for radial junction Si/a-Si:H NW solar cells. The p-i-n devices comprise dense and randomly oriented NWs with a p-doped cSi core and an a-Si:H absorption layer grown by PECVD on glass substrates. The impact of the ITO thickness and the Ag NW density on the solar cell performance are explored in detail. The conversion efficiency of the Si/a-Si:H NW solar cell is improved from $4.3 \%$ for the ITO-only contact (reference device) to $6.6 \%$ for the optimal hybrid contact. We show how the combinational use of Ag NWs and ITO provides supplementary degrees of freedom for the best trade-off between the optical and electrical properties of the contact to a NW solar cell. The developed hybrid contact is also suited for other NW optoelectronic devices with a challenging morphology, i.e. based on a dense array of non-vertical NWs with a core-shell structure.

\section{Methods and materials}

The solar cells consist of radial p-i-n junction NWs grown by plasma-enhanced chemical vapor deposition (PECVD) on a glass substrate ( $1 \times 1$ inch square). During the fabrication process, $\mathrm{p}$-doped Si NWs (ca. $40 \mathrm{~nm}$ middle-length diameter and $0.8 \mu \mathrm{m}$ length) were grown on a FTO (fluorine-doped tin oxide) layer on Corning glass using the vapor-liquid-solid method.(46) Metallic tin obtained from the reduction of the substrate oxide with $\mathrm{H}_{2}$ plasma was used as a catalyst. A p-doped a-Si:H layer with a doping gradient (10 
$\mathrm{nm})$, an intrinsic a-Si:H layer (100 nm) and an n-doped a-Si:H layer with a doping gradient $(10 \mathrm{~nm})$ were then deposited. The whole process from the $\mathrm{SnO}_{2}$ reduction until the $\mathrm{p}$-doped layer deposition was done in the same PECVD chamber during the single pump-down process. Further details about the NW growth can be found in (47).

A top ITO contact was sputtered on the NWs. A shadow mask was used during the magnetron sputtering to define circular solar cells with a $4 \mathrm{~mm}$ diameter. ITO properties were assessed with a reference layer deposited on a glass substrate using the same conditions. With the applied deposition conditions, a flat film of $80 \mathrm{~nm}$ displayed a sheet resistance $\left(R_{\square}\right)$ of $50 \Omega / \square$ after annealing $\left(240{ }^{\circ} \mathrm{C}, 20 \mathrm{~min}\right.$, air).

Suspensions of silver nanowires (10 $\mu \mathrm{m}$ long and $60 \mathrm{~nm}$ diameter) in isopropanol (IPA) were purchased from Sigma Aldrich. The initial concentration was $5 \mathrm{mg} / \mathrm{mL}$; the suspensions were further diluted in IPA with final concentrations ranging from $20 \%$ to $100 \%$ of the initial one. Comparison between different $\mathrm{Ag}$ NW densities was done using different solar cells on the same substrate separated by individual ITO pads Therefore, all cells experienced the same NW growth, ITO deposition and annealing conditions. A $2 \mu \mathrm{L}$ drop of Ag NW suspension was deposited on the cell at room temperature with a micropipette (1-10 $\mu \mathrm{L})$. The sample was annealed in an oven under ambient atmosphere at $240{ }^{\circ} \mathrm{C}$ for $20 \mathrm{~min}$. The annealing step is crucial, since it hugely decreases the overall sheet resistance, by improving the contact at the junctions between Ag NWs (welding and removing organic residues), without affecting the optical properties significantly. $(29,48)$ Although a degradation of silver nanowires may be expected with annealing in air, SEM observations did not show any change in the nanowires except at the junctions and electrical measurements on a network of Ag NWs on glass showed a decrease in the resistance just after annealing. The neighboring cells were mechanically separated with a diamond pen to prevent any electrical connection. The density of Ag nanowires was estimated from the dilution factor, the drop volume and the covered area. Scanning electron microscopy (SEM) observations were carried out with a Hitachi SU-8000 
field emission and a secondary electron detector. Accelerating voltages were around $1-3 \mathrm{kV}$ and working distances ranged from $4.5 \mathrm{~mm}$ to $11.9 \mathrm{~mm}$.

The performance of the solar cells was assessed using AAA solar simulator from Newport with standard AM1.5G illumination, calibrated with a certified c-Si cell. To avoid any possible change of the effective solar cell area due to the spreading of $\mathrm{Ag} \mathrm{NWs,} \mathrm{an} \mathrm{optical} \mathrm{mask} \mathrm{(} 3 \mathrm{~mm}$ diameter) was used to keep the illuminated area constant. A dot of Ag paint enabled good contact between the cell and the probe. The series resistance of each cell was determined with the slope of the I-V characteristics under illumination between $0.9 \mathrm{~V}$ and $1 \mathrm{~V}$. We used the Oriel IQE-200 setup for external quantum efficiency (EQE) measurements (composed of a monochromator and a lock-in amplifier).

\section{Results and discussion}

\section{Limited performance of ITO as a single front-contact material}

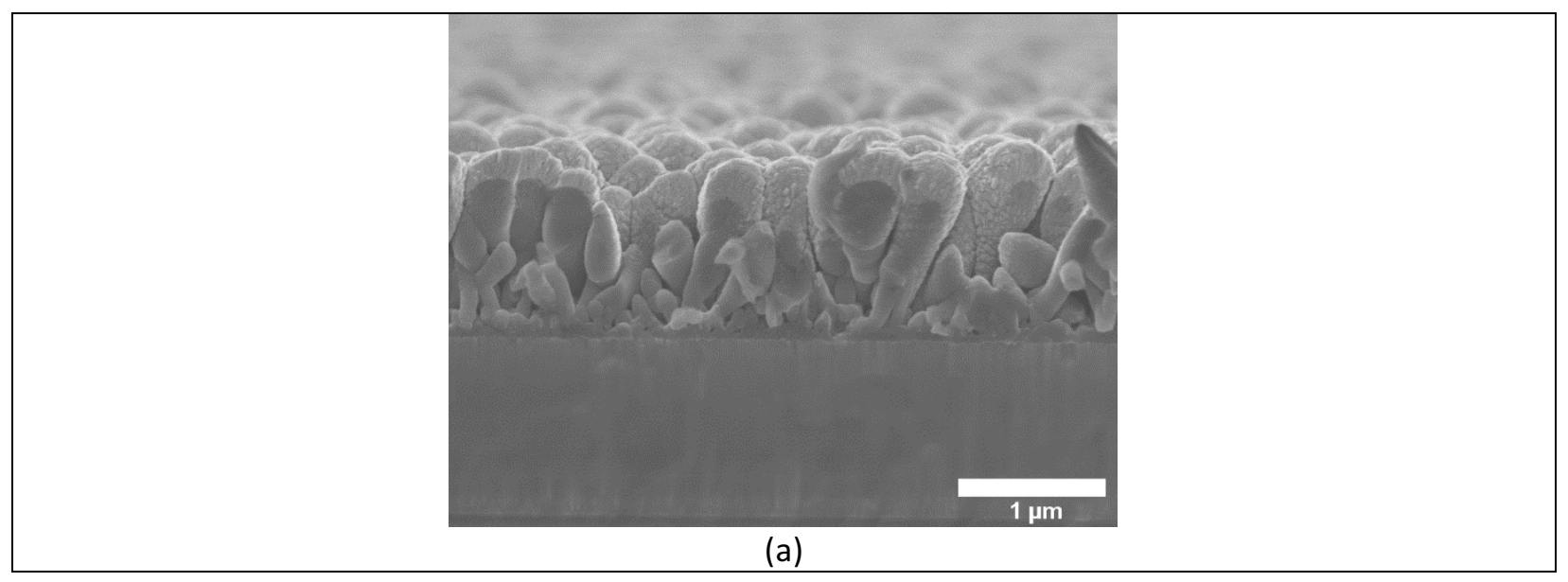




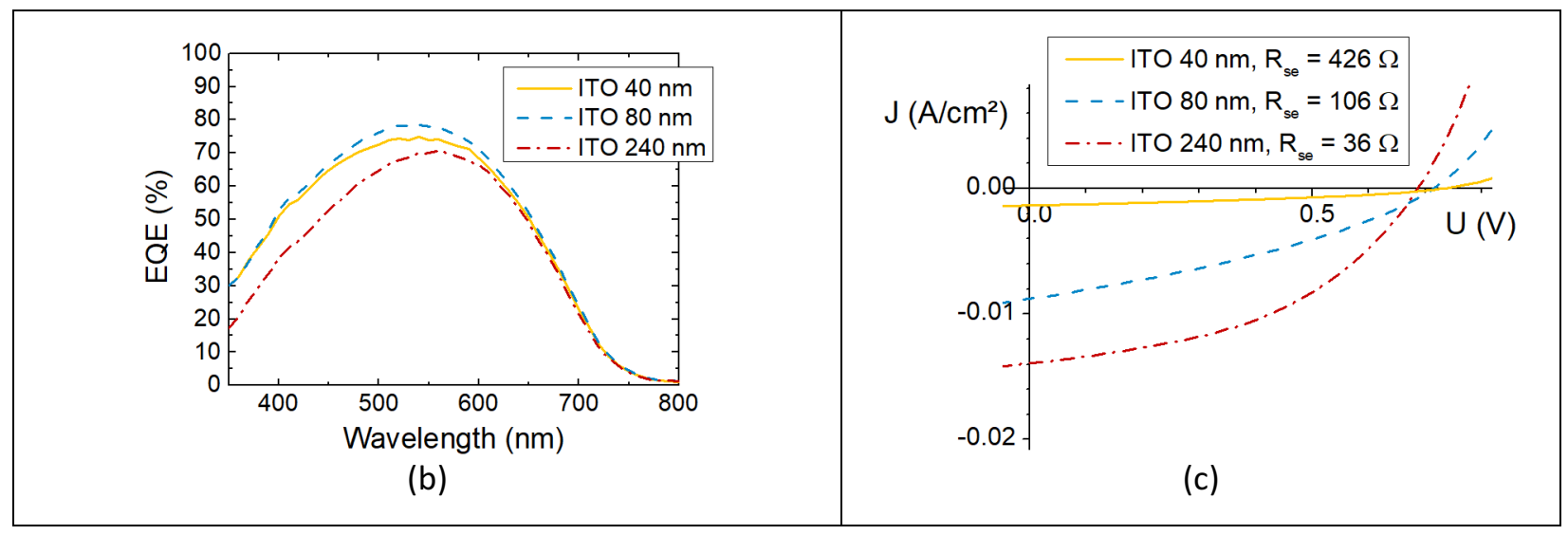

Figure 1: (a) Cross-sectional SEM image of a Si/a-Si:H NW solar cell with a $240 \mathrm{~nm}$ ITO top contact, (b) EQE measurements and (c) corresponding J-V curves under 1 Sun illumination for cells with different ITO thicknesses. The measured series resistances are indicated for each cell.

At first, we have analyzed a transparent top contact based exclusively on a sputtered ITO layer. Preliminary results suggest that the best conversion efficiency is obtained with a 7 min ITO deposition,(18) which corresponds to $240 \mathrm{~nm}$ ITO on a flat glass substrate yielding $R_{\square}=16 \Omega / \square$. As displayed in Fig. $1 \mathrm{a}$, the sputtered ITO is a thick layer on the top of the Si/a-Si:H NWs and coalesces between the neighboring NWs. This coalescence indeed helps to minimize the in-plane resistance of the electrode. It is also clear that the ITO coverage along the Si/a-Si:H NWs is not conformal, with very little ITO present at the bottom of the Si NWs.

Although $240 \mathrm{~nm}$ ITO was preliminarily shown to exhibit the best conversion efficiency, it is not the optimal thickness regarding the optical transmission into the cells, as will be explained in the following. Si/a-Si:H NW solar cells having the same p-i-n structure with different thicknesses of ITO were fabricated and characterized with EQE and J-V measurements, as seen in Fig. $1 \mathrm{~b}$ and $1 \mathrm{c}$. The nominal thickness of the ITO layer, as determined from reference samples on a flat glass substrate, equals to $240 \mathrm{~nm}, 80 \mathrm{~nm}$ and 40 $\mathrm{nm}$, respectively. It is important to note that the deposition of ITO is greatly altered when grown on a NW substrate, so that measurements of the contact transparency and conductivity on a flat glass substrate do 
not provide accurate information about these quantities on a real device. The assessment should be done directly on Si NWs despite the fact that it is difficult to disentangle the electrical and optical properties in this case. Nevertheless, EQE measurements help to probe the contact transmittance, since the very low illumination used in the set-up and the low resulting current make it almost insensitive to the differences in the contact resistance.

As shown in Fig. $1 \mathrm{~b}$, due to reflection and residual absorption of ITO,(49) the $240 \mathrm{~nm}$ ITO layer exhibits major optical losses in the visible range of the solar spectrum, as evidenced by its lowest EQE values. The most significant improvement occurs in the shorter wavelengths ( $350 \mathrm{~nm}-520 \mathrm{~nm}$ ), where the EQE values of the $80 \mathrm{~nm}$ ITO are higher than that of $240 \mathrm{~nm}$ ITO by more than 10\%. Despite the optical thickness and hence the absorption path twice as long, $80 \mathrm{~nm}$ ITO shows slightly higher EQE values than $40 \mathrm{~nm}$ ITO, with a maximal difference around $550 \mathrm{~nm}$. It can be understood with the antireflection effect of the ITO. As mentioned above, NW arrays trap the light much better than thin films, however, the reflection losses are still present.(8) ITO acts as an antireflection coating thanks to its intermediate refractive index (ca. 2.1 for ITO (49) compared to 3.6 for a-Si:H (50) at $550 \mathrm{~nm}$ ) and to its thickness. To maximize the transmittance at the peak of the solar spectrum (which also corresponds to a strong absorption for amorphous Si), the optimal thickness of ITO for flat cells was reported to be around $80 \mathrm{~nm}$.(51) Among the values we measured, $80 \mathrm{~nm}$ ITO is also clearly found to be the optimal thickness on top of nanowire ensembles.

Despite the best optical transmittance for $80 \mathrm{~nm}$ ITO (evidenced by its highest EQE values), it does not function as the most efficient top contact for the solar cell. As one sees in Fig. 1c, the solar cell with $80 \mathrm{~nm}$ ITO greatly suffers from low $\mathrm{J}_{\mathrm{sc}}$ and $\mathrm{FF}$, suggesting poor charge collection. The short-circuit current density under 1 Sun illumination is strongly reduced by a high series resistance due to the ITO contact. Because of the non-conformal deposition on NWs, the layer of ITO at the bottom of radial junctions is too thin, and the resistance of the contact greatly increases ( 3 times higher than that of $240 \mathrm{~nm}$ ITO). A thicker layer of ITO solves this problem. As a consequence, the solar cell implementing $240 \mathrm{~nm}$ ITO displays the lowest 
series resistance (see Fig. 1c) and thus the highest $\mathrm{J}_{\mathrm{sc}}$. However, the corresponding EQE values being lowest suggests major optical losses. A good compromise of the electrical conductivity and optical transmittance of the top contact is thus critical to improve the performance of the solar cell.

The non-conformal coverage of ITO is related to both the sputtering deposition method and the morphology of the Si NW array (dense array of disordered NWs with high aspect ratios). We note that for sparser arrays of core/shell NWs, the minimal thickness of sputtered ITO needed for a continuous layer is lower,(52) in which case the high optical transparency of the contact can be maintained without compromising the resistance. For dense NW arrays, instead of sputtering, deposition techniques enabling a more conformal growth such as atomic layer deposition (ALD) can be used.(53) Nonetheless, the total path that a charge carrier has to follow with an ideally conformal contact would still be much longer than the top-to-top distance (ca. 5 times for our NW morphology). This increases the contact resistance on a large scale. Therefore, a strategy to ensure high optical transparency and good electrical conductivity of the top contact is needed to improve the conversion efficiency of solar cells based on high aspect ratio NWs.

\section{Optimized ITO/Ag NWs hybrid contact}

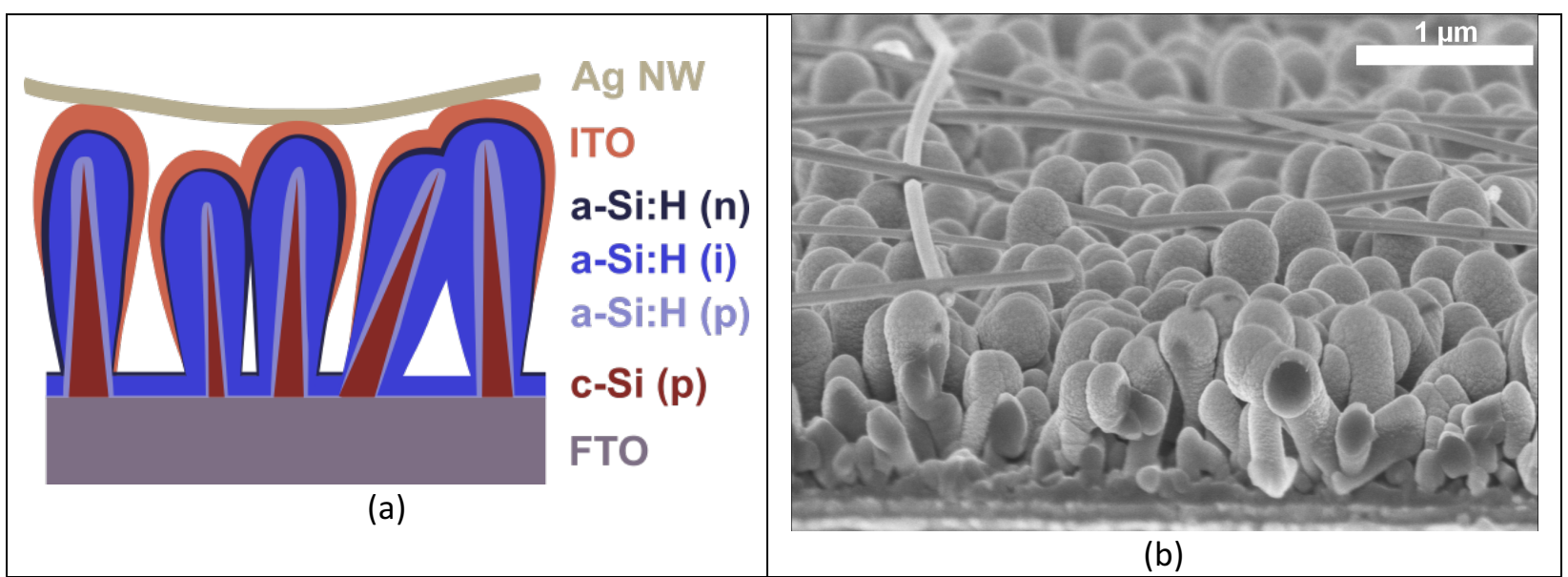


Figure 2: (a) Schematic of the Si/a-Si:H NW solar cell with the hybrid top contact made of ITO and Ag NWs, (b) cross-sectional SEM image of a similar Si/a-Si:H NW solar cell with a hybrid contact made of 80 $\mathrm{nm}$ thick ITO and Ag NWs.

In order to achieve high electrical conductivity while maintaining the high optical transparency, we propose to combine $80 \mathrm{~nm}$ ITO with Ag NWs. A schematic illustration of a Si/a-Si:H solar cell implementing such hybrid electrode is given in Figure $2 a$ while Figure $2 b$ shows the cross-sectional SEM image of a real cell (with similar structure) employing the hybrid top contact. In such a hybrid structure, the network of Ag NWs lying atop of the array offers an alternative current path to ITO, acting as a grid (Fig. 3a). This path is direct and at the same time very conductive because of the low resistivity of silver compared to ITO (ca. $2 \times 10^{-8} \Omega . m$ for a single Ag NW (54) vs. $4 \times 10^{-6} \Omega . m$ for the $80 \mathrm{~nm}$ reference layer of ITO deposited on a flat substrate).
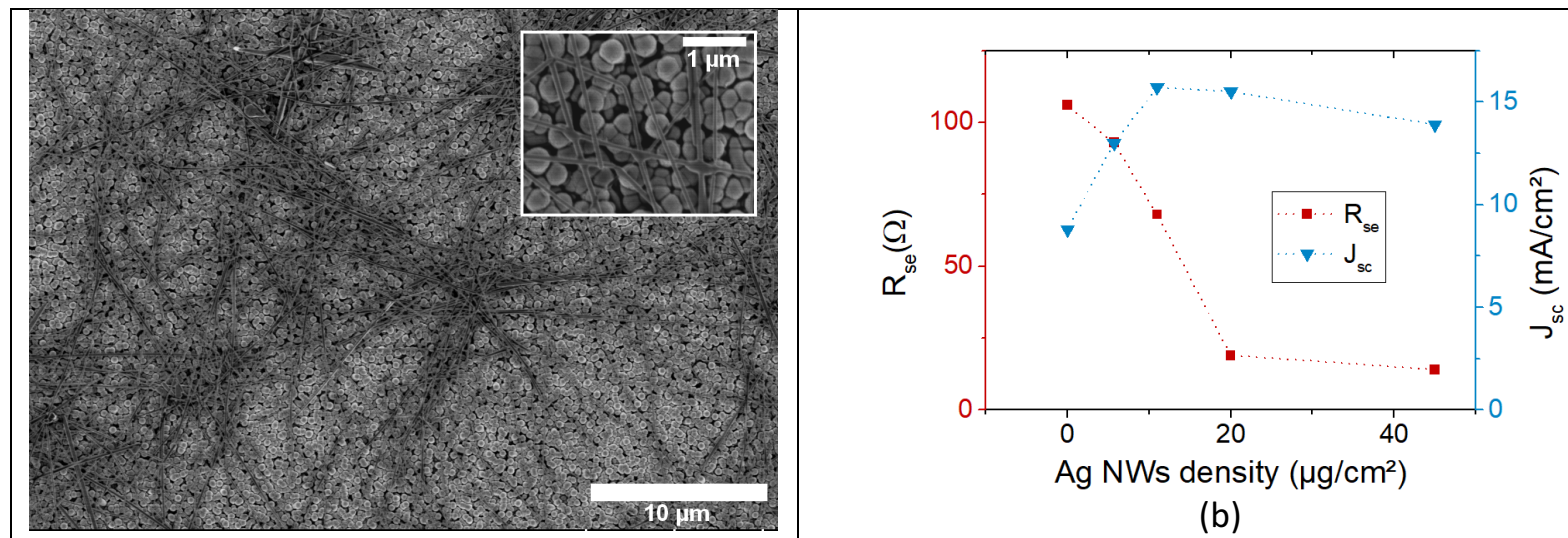

(a)

(b)

Figure 3: (a) Top-view SEM image of a Si NW solar cell with ITO and Ag NWs after annealing (ITO is 240 $\mathrm{nm}$ thick, the nominal Ag NWs density is around $37 \mu \mathrm{g} / \mathrm{cm}^{2}$ ), (b) series resistance and short-circuit current density for a cell with a hybrid contact consisting of $80 \mathrm{~nm}$ ITO and different densities of Ag NWs (all data acquired under 1 Sun). 
The conductivity of Ag NWs networks is closely related to the network densities (as detailed in the introduction), it is thus imperative to determine the optimal density of Ag NWs to be used in the hybrid contact. Therefore, Si/a-Si:H NW solar cells implementing $80 \mathrm{~nm}$ ITO combined with Ag NW networks of different densities were fabricated and characterized. The series resistance and the short-circuit current density under 1 Sun are plotted as a function of the density of Ag NWs in Fig. 3b. Significant reduction in the series resistance is achieved in the solar cell with a dense network of Ag NWs (six times lower than that in the same type of solar cell without Ag NWs). More specifically, the series resistance first decreases for denser Ag NW networks, then becomes steady at around $20 \Omega$ for a density above $20 \mu \mathrm{g} / \mathrm{cm}^{2}$. Adding more Ag NWs does not seem to further improve the electrical properties of the cell. The series resistance of the complete cell is an equivalent resistance, including the resistance of the NW core and a-Si:H layers, the contact resistance between materials, and the in-plane resistances of the top and back contacts. The decrease of series resistance with increasing Ag NW density suggests that the series resistance of the cell is mainly governed by the in-plane resistance of the top contact. This is indeed the only parameter contributing to the equivalent resistance which is sensitive to the change of density of the deposited $\mathrm{Ag}$ NWs. Compared to the expected percolation threshold for Ag NWs (calculated to be $1.7 \mu \mathrm{g} / \mathrm{cm}^{2}$ following eq. (1)), the experimentally determined density required for optimal cell operation is higher. The first reason is an overestimation of the experimental density, for instance because of the drying ring. Moreover, reaching percolation means that one conductive path exists but its resistance can still be high. Thus, a percolating network does not necessarily result in a good conduction.(55) A much higher Ag NW density is expected to be used in optoelectronic devices which require a particularly low resistance such as solar cells.

Figure $3 \mathrm{~b}$ shows the evolution of the short-circuit current density as a function of Ag NW densities. Two opposing effects occur. Because of the reflection and absorption by Ag NWs, less light is transmitted into the cell (i.e. lower absorption) and a decrease of $\mathrm{J}_{\mathrm{sc}}$ can be expected. Yet $\mathrm{Ag} \mathrm{NWs}$ also reduce the series 
resistance. They improve the collection of charge carriers, which would result in a higher $\mathrm{J}_{\mathrm{sc}}$. At low Ag NW densities $\left(\leq 11 \mu \mathrm{g} / \mathrm{cm}^{2}\right)$, J $\mathrm{sc}$ quickly increases with increasing Ag NW density. It demonstrates that the shortcircuit current density was limited by the series resistance of the cell. Above $11 \mu \mathrm{g} / \mathrm{cm}^{2}$, however, $\mathrm{J}_{\mathrm{sc}}$ is seen to slowly decrease with further increase of the Ag NW density. With a higher Ag NW density, the transparency loss is more important than the gain by reduction in series resistance. The moderate steepness of the $J_{s c}$ decrease, compared to its increase, highlights the important impact of the series resistance on the $J_{s c}$ value. A density close to $20 \mu \mathrm{g} / \mathrm{cm}^{2}$ with $80 \mathrm{~nm}$ ITO gives the best compromise with a good collection of charge carriers and a low amount of light absorbed or reflected by the contact. As a result, an increased $\mathrm{J}_{\mathrm{sc}}$ is achieved with respect to the reference cell with $240 \mathrm{~nm}$ ITO alone.

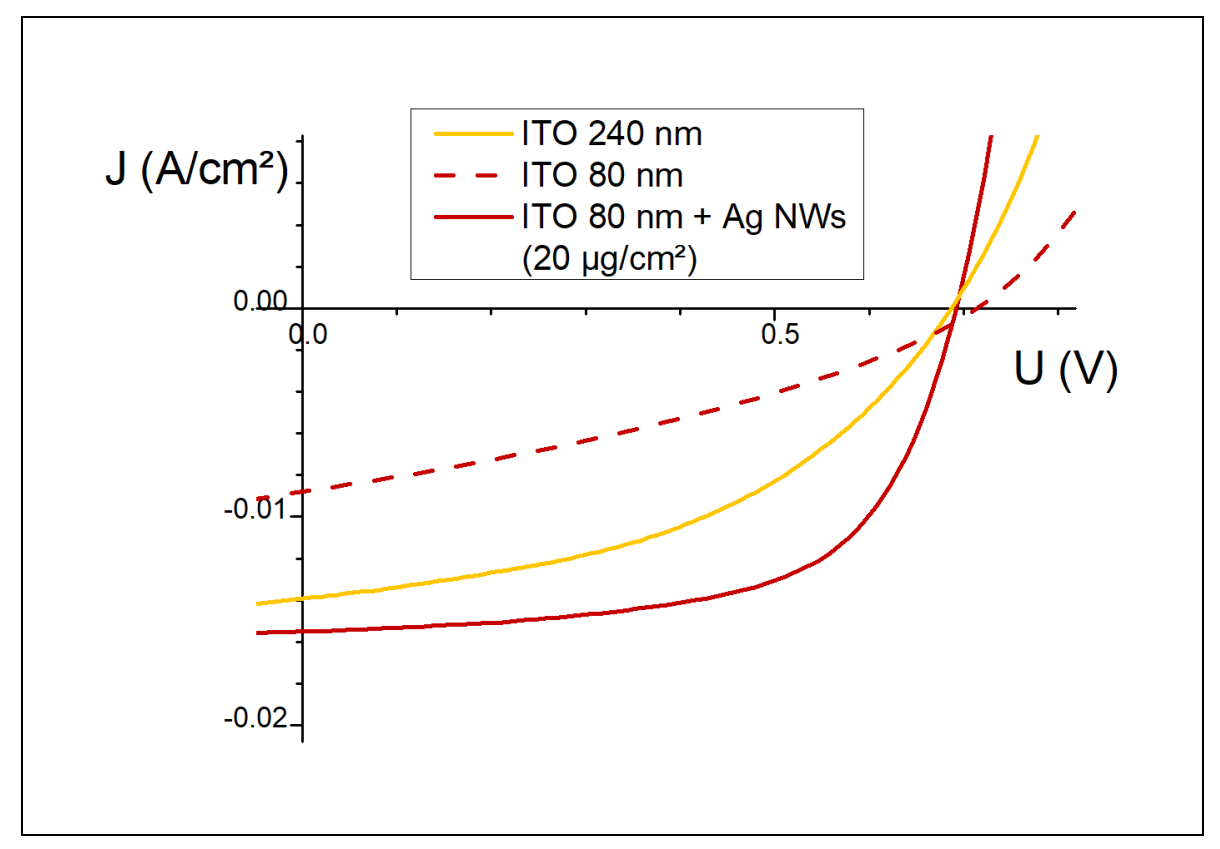

Figure 4: J-V measurements under 1 Sun for the reference top contact of $240 \mathrm{~nm}$ ITO, the optimized ITO thickness of $80 \mathrm{~nm}$ and the optimized hybrid ITO/Ag NWs contact.

Table 1: Characteristics of cells with $240 \mathrm{~nm}$ ITO (reference) and $80 \mathrm{~nm}$ ITO with various Ag NWs densities (measurements under 1 Sun). Lines in bold font correspond to the cells presented in Fig. 4. 


\begin{tabular}{|c|c|c|c|c|c|}
\hline $\begin{array}{l}\text { ITO nominal } \\
\text { thickness } \\
\text { (nm) }\end{array}$ & $\begin{array}{c}\text { Ag NWs } \\
\text { density } \\
\left(\mu \mathrm{g} / \mathrm{cm}^{2}\right)\end{array}$ & $\begin{array}{c}J_{s c} \\
\left(\mathrm{~mA} / \mathrm{cm}^{2}\right)\end{array}$ & $\begin{array}{l}V_{\text {oc }} \\
\text { (V) }\end{array}$ & $\begin{array}{l}\text { FF } \\
(\%)\end{array}$ & $\begin{array}{c}\text { Efficiency } \\
\text { (\%) }\end{array}$ \\
\hline 240 & - & 14.0 & 0.68 & 45 & 4.3 \\
\hline 80 & - & 8.8 & 0.71 & 34 & 2.1 \\
\hline 80 & 5.7 & 13.0 & 0.72 & 35 & 3.3 \\
\hline 80 & 11 & 15.7 & 0.71 & 54 & 6.0 \\
\hline 80 & 20 & 15.5 & 0.69 & 62 & 6.6 \\
\hline 80 & 45 & 13.9 & 0.71 & 64 & 6.3 \\
\hline
\end{tabular}

The improvement of the solar cell performance was assessed with J-V measurements under AM1.5 illumination. Figure 4 displays the J-V measurements for the reference cell ( $240 \mathrm{~nm}$ ITO), the cell with the optically optimal layer of ITO (80 nm ITO) and the cell with the hybrid contact for which the maximal conversion efficiency is achieved (ITO $80 \mathrm{~nm}$ and $20 \mu \mathrm{g} / \mathrm{cm}^{2} \mathrm{Ag} \mathrm{NWs}$ ). The characteristics of all cells are summarized in Table 1, including those with other densities of Ag NWs on $80 \mathrm{~nm}$ ITO.

When decreasing the ITO thickness from $240 \mathrm{~nm}$ to $80 \mathrm{~nm}$, the $J_{s c}$ value decreases from $14.0 \mathrm{~mA} / \mathrm{cm}^{2}$ to $8.0 \mathrm{~mA} / \mathrm{cm}^{2}$ despite the gain in transmittance. This is due to the poor collection of charge carriers. As detailed above, low densities of Ag NWs induce a better collection, with no major optical losses. The higher $J_{s c}$ obtained with the optimized hybrid contact compared to the reference confirms that the short-circuit current is limited by ITO absorption for $240 \mathrm{~nm}$ thick ITO, as was expected from EQE measurements. When the thickness of ITO is reduced and the series resistance is low enough to efficiently collect charge carriers, $\mathrm{J}_{\mathrm{sc}}$ increases from $14.0 \mathrm{~mA} / \mathrm{cm}^{2}$ to $15.5 \mathrm{~mA} / \mathrm{cm}^{2}$.

As seen from Table 1, the open-circuit voltage values remain rather similar for all analyzed cells (variation is below 6\%). Small variations of $\mathrm{V}_{\mathrm{OC}}$ were observed before the deposition of $\mathrm{Ag} \mathrm{NWs}$, therefore they are not related to the quantity of Ag NWs, but probably stem from the inhomogeneity of the Si NW growth. The obtained $\mathrm{V}_{\text {oc }}$ is typical of radial junction Si NW solar cells.(52) The sensitivity of the open-circuit voltage 
to the NW dimensions and density may account for the difference with values reported for this structure of Si/a-Si:H NW solar cells (0.79 V). (47)

In addition, not only $\mathrm{J}_{\mathrm{sc}}$ is increased when using $\mathrm{Ag} \mathrm{NWs,} \mathrm{but} \mathrm{the} \mathrm{fill} \mathrm{factor} \mathrm{is} \mathrm{also} \mathrm{strongly} \mathrm{improved.} \mathrm{As} \mathrm{the}$ solar cell efficiency $\eta$ can be calculated as

$$
\eta=\frac{\mathrm{J}_{\mathrm{sc}} \mathrm{V}_{\mathrm{oc}} \mathrm{FF}}{\mathrm{P}}
$$

with $\mathrm{P}$ the light power (1000 W/cm² for 1 Sun illumination), the fill factor has a large impact on the solar cell performance. As a consequence, compared to the cell with the reference top electrode, the conversion efficiency substantially increases from $4.3 \%$ (reference cell) to $6.6 \%$ (hybrid contact).

The fill factor (FF) is related to the collection of charge carriers and can be altered by the series and shunt resistances of the solar cell and the recombinations of charge carriers. The observed improvement in FF clearly originates from the series resistance decrease. While the shunt resistance can have a significant impact in radial junction Si/a-Si:H solar cells, it should not be affected by the deposition of Ag NWs (SEM observations show that Ag NWs do not contact the bottom of the solar cell). Besides, non-radiative recombinations depend mainly on the surface recombination velocity and the presence of defects in the Si/a-Si:H NWs. Therefore, they are not expected to change with the Ag NW deposition. As the series resistance decreases with higher Ag NWs densities, the fill factor is improved, which demonstrates again that the maximal power of the cell was limited by the resistance of the top contact. The use of a hybrid contact enables us to reach a performance that was not possible with a single layer of TCO, because the composite separates the optical and electrical optimizations. It results in a short- and a long-range collection of the charge carriers. We note that this consideration remains valid for other contact materials and devices, such as AZO for ZnO/a-Si:H NW solar cells.(34) 


\section{Conclusion}

A hybrid top electrode is demonstrated to be beneficial for solar cells containing high density non-vertical radial junction Si/a-Si:H NW arrays. The implementation of Ag NWs largely compensates the poor charge carrier collection of the thin ITO layer, making it a highly transparent and conductive top contact ideally suited to Si NWs solar cells. The best conversion efficiency was achieved for a hybrid contact consisting of $80 \mathrm{~nm}$ ITO with Ag NWs on top (nominal density around $20 \mu \mathrm{g} / \mathrm{cm}^{2}$ ), annealed in air. Thanks to a better collection of electrons and higher overall transparency when compared to the thick ITO-only contact, both

$\mathrm{J}_{\mathrm{sc}}$ and $\mathrm{FF}$ are increased, resulting in a significant improvement in conversion efficiency from $4.3 \%$ to $6.6 \%$. The demonstrated hybrid contact could be used also in other types of NW devices including applications requiring mechanical flexibility, as the thinner ITO and flexible Ag NW network exhibit better robustness against the mechanical stress. 


\section{Acknowledgement}

This work has been financially supported by EU H2020 ERC project "NanoHarvest" (grant no. 639052).

The authors would like to thank Stéphane Collin for his help for the EQE measurements. 


\section{References}

1. De S, Higgins TM, Lyons PE, Doherty EM, Nirmalraj PN, Blau WJ, et al. Silver Nanowire Networks as Flexible, Transparent, Conducting Films: Extremely High DC to Optical Conductivity Ratios. ACS Nano. 2009 Jul 28;3(7):1767-74.

2. Zhang Y, Liu H. Nanowires for High-Efficiency, Low-Cost Solar Photovoltaics. Crystals. 2019 Feb 8;9(2):87.

3. Chen J, Wang K, Hartman L, Zhou W. $\mathrm{H}_{2} \mathrm{~S}$ Detection by Vertically Aligned CuO Nanowire Array Sensors. J Phys Chem C. 2008 Oct 16;112(41):16017-21.

4. Lai E, Kim W, Yang P. Vertical nanowire array-based light emitting diodes. Nano Res. 2008 Aug;1(2):123-8.

5. Larrieu G, Han X-L. Vertical nanowire array-based field effect transistors for ultimate scaling. Nanoscale. 2013;5(6):2437.

6. Cohin Y, Mauguin O, Largeau L, Patriarche G, Glas F, Søndergård E, et al. Growth of Vertical GaAs Nanowires on an Amorphous Substrate via a Fiber-Textured Si Platform. Nano Lett. 2013 Jun $12 ; 13(6): 2743-7$.

7. Al-Ghzaiwat M, Foldyna M, Fuyuki T, Chen W, Johnson EV, Meot J, et al. Large Area Radial Junction Silicon Nanowire Solar Mini-Modules. Sci Rep. 2018 Dec;8(1):1651.

8. Zhu J, Yu Z, Burkhard GF, Hsu C-M, Connor ST, Xu Y, et al. Optical Absorption Enhancement in Amorphous Silicon Nanowire and Nanocone Arrays. Nano Lett. 2009 Jan 14;9(1):279-82.

9. Jung J-Y, Guo Z, Jee S-W, Um H-D, Park K-T, Lee J-H. A strong antireflective solar cell prepared by tapering silicon nanowires. Opt Express. 2010 Sep 13;18(S3):A286.

10. Lin XX, Hua X, Huang ZG, Shen WZ. Realization of high performance silicon nanowire based solar cells with large size. Nanotechnology. 2013 Jun 14;24(23):235402.

11. Garnett E, Yang P. Light Trapping in Silicon Nanowire Solar Cells. Nano Lett. 2010 Mar 10;10(3):1082-7.

12. Sivakov V, Andrä G, Gawlik A, Berger A, Plentz J, Falk F, et al. Silicon Nanowire-Based Solar Cells on Glass: Synthesis, Optical Properties, and Cell Parameters. Nano Lett. 2009 Apr 8;9(4):1549-54.

13. Tian B, Zheng $X$, Kempa TJ, Fang $Y, Y u ~ N, ~ Y u ~ G$, et al. Coaxial silicon nanowires as solar cells and nanoelectronic power sources. Nature. 2007 Oct;449(7164):885-9.

14. Yu P, Wu J, Liu S, Xiong J, Jagadish C, Wang ZM. Design and fabrication of silicon nanowires towards efficient solar cells. Nano Today. 2016 Dec;11(6):704-37.

15. Haug F-J, Ballif C. Light management in thin film silicon solar cells. Energy Environ Sci. 2015;8(3):82437. 
16. Matsui T, Maejima K, Bidiville A, Sai H, Koida T, Suezaki T, et al. High-efficiency thin-film silicon solar cells realized by integrating stable a-Si:H absorbers into improved device design. Jpn J Appl Phys. 2015 Aug 1;54(8S1):08KB10.

17. Misra S, Yu L, Foldyna M, Roca i Cabarrocas P. High efficiency and stable hydrogenated amorphous silicon radial junction solar cells built on VLS-grown silicon nanowires. Sol Energy Mater Sol Cells. 2013 Nov;118:90-5.

18. Misra S. Single and tandem radial junction silicon thin film solar cells based on PECVD grown crystalline silicon nanowire arrays. Ecole polytechnique; 2015.

19. Adachi MM, Anantram MP, Karim KS. Core-shell silicon nanowire solar cells. Sci Rep. 2013 Dec;3(1):1546.

20. Xie X, Zeng X, Yang P, Li H, Li J, Zhang X, et al. Radial n-i-p structure SiNW-based microcrystalline silicon thin-film solar cells on flexible stainless steel. Nanoscale Res Lett. 2012 Dec;7(1):621.

21. Misra S, Yu L, Foldyna M, Roca i Cabarrocas P. New Approaches to Improve the Performance of ThinFilm Radial Junction Solar Cells Built Over Silicon Nanowire Arrays. IEEE J Photovolt. 2015 Jan;5(1):40-5.

22. Jeong S, Garnett EC, Wang S, Yu Z, Fan S, Brongersma ML, et al. Hybrid Silicon Nanocone-Polymer Solar Cells. Nano Lett. 2012 Jun 13;12(6):2971-6.

23. Kim YH, Sachse C, Machala ML, May C, Müller-Meskamp L, Leo K. Highly Conductive PEDOT:PSS Electrode with Optimized Solvent and Thermal Post-Treatment for ITO-Free Organic Solar Cells. Adv Funct Mater. 2011 Mar 22;21(6):1076-81.

24. Bae S, Kim H, Lee $Y, X u X$, Park J-S, Zheng Y, et al. Roll-to-roll production of 30-inch graphene films for transparent electrodes. Nat Nanotechnol. 2010 Aug;5(8):574-8.

25. Cai W, Zhu Y, Li X, Piner RD, Ruoff RS. Large area few-layer graphene/graphite films as transparent thin conducting electrodes. Appl Phys Lett. 2009 Sep 21;95(12):123115.

26. Rowell MW, Topinka MA, McGehee MD, Prall H-J, Dennler G, Sariciftci NS, et al. Organic solar cells with carbon nanotube network electrodes. Appl Phys Lett. 2006 Jun 5;88(23):233506.

27. Hu L, Kim HS, Lee J-Y, Peumans P, Cui Y. Scalable Coating and Properties of Transparent, Flexible, Silver Nanowire Electrodes. ACS Nano. 2010 May 25;4(5):2955-63.

28. Jang H, Kim D, Tak H, Nam J, Kim T. Ultra-mechanically stable and transparent conductive electrodes using transferred grid of Ag nanowires on flexible substrate. Curr Appl Phys. 2016 Jan;16(1):24-30.

29. Lee J-Y, Connor ST, Cui Y, Peumans P. Solution-Processed Metal Nanowire Mesh Transparent Electrodes. Nano Lett. 2008 Feb;8(2):689-92.

30. Langley D, Giusti G, Mayousse C, Celle C, Bellet D, Simonato J-P. Flexible transparent conductive materials based on silver nanowire networks: a review. Nanotechnology. $2013 \mathrm{Nov}$ 15;24(45):452001. 
31. De S, King PJ, Lyons PE, Khan U, Coleman JN. Size Effects and the Problem with Percolation in Nanostructured Transparent Conductors. ACS Nano. 2010 Dec 28;4(12):7064-72.

32. Lagrange M, Langley DP, Giusti G, Jimenez C, Bréchet Y, Bellet D. Optimization of silver nanowirebased transparent electrodes: effects of density, size and thermal annealing. :16.

33. Bergin SM, Chen Y-H, Rathmell AR, Charbonneau P, Li Z-Y, Wiley BJ. The effect of nanowire length and diameter on the properties of transparent, conducting nanowire films. Nanoscale.

2012;4(6):1996.

34. Pathirane MK, Hosseinzadeh Khaligh H, Goldthorpe IA, Wong WS. Al-doped ZnO/Ag-nanowire Composite Electrodes for Flexible 3-Dimensional Nanowire Solar Cells. Sci Rep [Internet]. 2017 Dec [cited 2019 Oct 22];7(1). Available from: http://www.nature.com/articles/s41598-017-07180-1

35. Hsiao S-T, Tien H-W, Liao W-H, Wang Y-S, Li S-M, MMa C-C, et al. A highly electrically conductive graphene-silver nanowire hybrid nanomaterial for transparent conductive films. J Mater Chem $C$. 2014 Jun 27;2(35):7284.

36. Lee M-S, Lee K, Kim S-Y, Lee H, Park J, Choi K-H, et al. High-Performance, Transparent, and Stretchable Electrodes Using Graphene-Metal Nanowire Hybrid Structures. Nano Lett. 2013 Jun 12;13(6):2814-21.

37. Crupi I, Boscarino S, Strano V, Mirabella S, Simone F, Terrasi A. Optimization of ZnO:Al/Ag/ZnO:Al structures for ultra-thin high-performance transparent conductive electrodes. Thin Solid Films. 2012 Apr;520(13):4432-5.

38. Kim TH, Kim CH, Kim SK, Lee YS, Park LS. High Quality Transparent Conductive ITO/Ag/ITO Multilayer Films Deposited on Glass Substrate at Room Temperature. Mol Cryst Liq Cryst. 2010 Oct 18;532(1):112/[528]-118/[534].

39. Reinhard M, Eckstein R, Slobodskyy A, Lemmer U, Colsmann A. Solution-processed polymer-silver nanowire top electrodes for inverted semi-transparent solar cells. Org Electron. 2013 Jan;14(1):2737.

40. Liu J, Yi Y, Zhou Y, Cai H. Highly Stretchable and Flexible Graphene/ITO Hybrid Transparent Electrode. Nanoscale Res Lett. 2016 Dec;11(1):108.

41. Kim A, Won Y, Woo K, Kim C-H, Moon J. Highly Transparent Low Resistance ZnO/Ag Nanowire/ZnO Composite Electrode for Thin Film Solar Cells. ACS Nano. 2013 Feb 26;7(2):1081-91.

42. Singh M, Rana TR, Kim S, Kim K, Yun JH, Kim J. Silver Nanowires Binding with Sputtered ZnO to Fabricate Highly Conductive and Thermally Stable Transparent Electrode for Solar Cell Applications. ACS Appl Mater Interfaces. 2016 May 25;8(20):12764-71.

43. Tsai W-C, Thomas SR, Hsu C-H, Huang Y-C, Tseng J-Y, Wu T-T, et al. Flexible high performance hybrid AZO/Ag-nanowire/AZO sandwich structured transparent conductors for flexible $\mathrm{Cu}(\mathrm{In}, \mathrm{Ga}) \mathrm{Se}{ }_{2}$ solar cell applications. J Mater Chem A. 2016;4(18):6980-8. 
44. Park J, Ouyang Z, Yan C, Sun K, Sun H, Liu F, et al. Hybrid Ag Nanowire-ITO as Transparent Conductive Electrode for Pure Sulfide Kesterite $\mathrm{Cu}_{2} \mathrm{ZnSnS}_{4}$ Solar Cells. J Phys Chem C. 2017 Sep 28;121(38):20597-604.

45. Turner-Evans DB, Emmer H, Chen CT, Atwater HA. Flexible, Transparent Contacts for Inorganic Nanostructures and Thin Films. Adv Mater. 2013 Aug 7;25(29):4018-22.

46. Wagner RS, Ellis WC. VAPOR-LIQUID-SOLID MECHANISM OF SINGLE CRYSTAL GROWTH. Appl Phys Lett. 1964 Mar;4(5):89-90.

47. Al-Ghzaiwat M, Foti A, Nuesslein A, Halagacka L, Meot J, Labouret A, et al. Toward Efficient Radial Junction Silicon Nanowire-Based Solar Mini-Modules. Phys Status Solidi RRL - Rapid Res Lett. 2019 Feb;13(2):1800402.

48. Hwang B, An Y, Lee H, Lee E, Becker S, Kim Y-H, et al. Highly Flexible and Transparent Ag Nanowire Electrode Encapsulated with Ultra-Thin Al2O3: Thermal, Ambient, and Mechanical Stabilities. Sci Rep. 2017 Feb;7(1):41336.

49. Yang $Y$, Sun $X W$, Chen $B J, X u C X$, Chen $T P$, Sun $C Q$, et al. Refractive indices of textured indium tin oxide and zinc oxide thin films. Thin Solid Films. 2006 Jul;510(1-2):95-101.

50. Swanepoel R. Determination of the thickness and optical constants of amorphous silicon. J Phys [E]. 1983 Dec;16(12):1214-22.

51. Pla J, Tamasi M, Rizzoli R, Losurdo M, Centurioni E, Summonte C, et al. Optimization of ITO layers for applications in a-Siyc-Si heterojunction solar cells. Thin Solid Films. 2003;8.

52. Sun $X$, Zhang $T$, Wang J, Yang F, Xu L, Xu J, et al. Firmly standing three-dimensional radial junctions on soft aluminum foils enable extremely low cost flexible thin film solar cells with very high powerto-weight performance. Nano Energy. 2018 Nov;53:83-90.

53. Coutancier D, Zhang S-T, Bernardini S, Fournier O, Mathieu-Pennober T, Donsanti F, et al. ALD of ZnO:Ti: Growth Mechanism and Application as an Efficient Transparent Conductive Oxide in Silicon Nanowire Solar Cells. ACS Appl Mater Interfaces. 2020 Apr 23;acsami.9b22973.

54. Bellew AT, Manning HG, Gomes da Rocha C, Ferreira MS, Boland JJ. Resistance of Single Ag Nanowire Junctions and Their Role in the Conductivity of Nanowire Networks. ACS Nano. 2015 Nov 24;9(11):11422-9.

55. De S, King PJ, Lyons PE, Khan U, Coleman JN. Size Effects and the Problem with Percolation in Nanostructured Transparent Conductors. ACS Nano. 2010 Dec 28;4(12):7064-72. 